\title{
Association Between Physical Activity, Menstrual Cycle Characteristics, and Body Weight in Young South Indian Females
}

\author{
Shabnam Omidvar ${ }^{\circledR}$, Fatemeh Nasiri Amiri², Mozhgan Firouzbakht ${ }^{3}$, Afsaneh Bakhtiari $^{4^{*}}{ }^{\circledR}$, Khyrunnisa $^{\circ}$ \\ Begum $^{5}$
}

\begin{abstract}
Objectives: Physical activity is considered as an essential component of a woman's ability to maintain or improve her level of wellness. Nevertheless, women, particularly young girls, usually pay less attention to health-promoting behaviors such as regular physical activity. Given the significance of physical activity in woman' health status, the present study aimed to determine the influence of such activity and body mass index (BMI), especially on menstrual characteristics and menarche age among young females according to their socioeconomic status (SES).

Materials and Methods: Data collection included two parts. First, a cross-sectional study was conducted on 1000 healthy and young females aged 11-28 years and standardized self-reporting questionnaires were used to obtain relevant information. Then, purposive sampling technique was utilized to compare the information pertinent to physically active and inactive females or those with sedentary behavior. The data were later analyzed using the chi-square test by SPSS 16.

Results: Based on the results, the majority of the young female population were physically inactive and only 121 women (12.1\%) enjoyed exercising at least for 30 minutes three times a week or more. In addition, there was a significant correlation between BMI, SES, and physical activity involvement. The cycle length, the regularity of periods, and the severity of dysmenorrhea exhibited a positive association with involvement in physical activity.

Conclusions: Overall, physical activity had a positive influence on menstrual characteristics in young females. Therefore, it is important to educate women for regular physical activity in order to modify or reduce menstrual cycle disturbances.

Keywords: Menstrual cycle, Physical activity, Menstruation characteristics
\end{abstract}

\section{Introduction}

Physical activity plays a key role in maintaining or improving an individual's wellness. Many healthassociated advantages are demonstrated regarding physical activity in adolescents (1). However, reports from European countries indicate that youths are too inactive to perform the suggested physical activity (2). Meanwhile, a report from developing countries is scarce.

Regular physical activity decreases the risk of chronic diseases (3). Studies have further found evidence that physical activity enhances respiratory, muscle, cardiovascular, and cognitive functions. Moreover, physical activity decreases the severity and occurrence of depression and stress level (4-9).

There are several suggestions emphasizing on a lifestyle which includes various activities ranging from walking briskly, climbing the stairs, doing run-of-the-mill household works to involving in activities with severe intensity $(10,11)$. All adults are recommended to engage in 30-minute daily activities with an intensity equal to 3-4 miles of walking per hour $(11,12)$, which seems to be realistic and achievable for the majority of the adult population. Previous research indicated that women's decision concerning physical activities relies on their attitudes in this regard (13).

On the other hand, menstrual cycles and related problems are always a matter of concern among women. Similarly, reports and reviews about menstruation and menstrual cycle confirmed that physical activities and exercises alleviative menstrual pain and other symptoms. Further, physical activity four hours per week or more is related to longer cycle length. Apparently, the longer duration of the cycles can have a relation with modifications in folliclestimulating hormone pulses during the phase transition. On the other hand, the longer cycle length is related to late ovulation and longer follicular phase since luteal phases have a fixed duration which is limited to 14 days (14).

Considering the above-mentioned discussions,

Received 5 December 2018, Accepted 11 March 2019, Available online 20 March 2019

${ }^{1}$ Social Determinants of Health Research Center, Health Research Institute, Babol University of Medical Sciences, Babol, I.R. Iran. ${ }^{2}$ Infertility and Health Reproductive Research Center, Health Research Institute, Babol University of Medical Sciences, Babol, I.R. Iran. ${ }^{3}$ Department of Midwifery, Babol Branch, Islamic Azad University, Babol, Iran. ${ }^{4}$ Mobility Impairment Research Center, Health Research Institute, Babol University of Medical Sciences, Babol, I.R.Iran. ${ }^{5}$ University of Mysore, Mysore, India.

*Corresponding Author: Afsaneh Bakhtiari, Tel: Tel: 09113116889, Email: afbakhtiari@gmail.com 
menstruation is regarded as a normal process in women at the reproductive age and any disturbance could be debilitating for a woman. Accordingly, it is considered quite worthwhile to explore the effect of physical activity on menstrual characteristics of such women.

\section{Methods and Materials}

The present study, using a cross-sectional design, was conducted in urban areas of Mysore in South India. A number of 1000 healthy, young, and mature female students aged 11-28 years formed the study population who volunteered to participate in the study by signing the written consent form. The aim of the study and the contents of the questionnaire were explained to each participant.

Then, based on the purpose of the study, the prevalence of physical activity involvement was assessed among the study population. A total of 121 physically active women (i.e., those who exercised three times or more in a week) were selected from the population applying a purposive sampling method. Then, 130 women, within the same age of physically active group, were opted for inclusion in the sedentary female group. Essential information was elicited from the selectees who completed the questionnaires related to demographic features and menstrual patterns. Next, the severity of dysmenorrhea in dysmenorrheic subjects was measured using the visual analogue scale which was developed by Revill et al (15). As suggested by Higham et al (16), to describe the degree to which the sanitary wear was soiled, information regarding menstrual bleeding was obtained by using a pictorial chart. Moreover, the research protocol was approved by the Ethics Committee of the University of Mysore.

The data were analyzed using SPSS software, version 16.0. Additionally, descriptive statistics were applied to determine the frequency of menstrual characteristics, the prevalence of dysmenorrhea, along with the severity of dysmenorrhea based on physical activity. Finally, the categorical data were analyzed utilizing the chi-square or Fisher exact test.

\section{Results}

Subjective information is presented in Table 1. The average age of the sample was $18.2 \pm 3.2$ years and the majority of subjects practiced Hinduism (84\%). Likewise, more than $85 \%$ of women were from a nuclear family with less than five members. In addition, a higher proportion of women (52.9\%) belonged to the middle level of socioeconomic status (SES) while only $10.4 \%$ of them were from high SES.

Most of the young females were physically inactive while only 121 women $(12.1 \%)$ were practicing exercises three times a week or more.

Table 2 demonstrates the pattern of the distribution of physical activity based on the participants' body mass index (BMI), SES, and age. As shown in Table 2, there is a significant association between BMI and SES with
Table 1. Demographic Information About Women

\begin{tabular}{|c|c|c|c|}
\hline Information & & No. & $\%$ \\
\hline \multirow{4}{*}{ Age (y) } & $11-15$ & 226 & 22.6 \\
\hline & $16-18$ & 252 & 25.2 \\
\hline & $19-21$ & 271 & 27.1 \\
\hline & $\geq 22$ & 251 & 25.1 \\
\hline \multirow{4}{*}{ Religion } & Hindu & 836 & 84.0 \\
\hline & Muslim & 123 & 12.3 \\
\hline & Christian & 24 & 2.4 \\
\hline & Other & 13 & 1.3 \\
\hline \multirow{4}{*}{ Education } & Up to 10 th standard & 469 & 47.0 \\
\hline & Pre-university & 332 & 33.2 \\
\hline & Graduation & 194 & 19.4 \\
\hline & Post-graduation & 4 & 0.4 \\
\hline \multirow{3}{*}{ Type of family } & Joint & 64 & 6.4 \\
\hline & Nuclear & 852 & 85.7 \\
\hline & Extended & 78 & 7.8 \\
\hline \multirow{3}{*}{ Family size } & $\leq 4$ & 538 & 53.9 \\
\hline & $5-8$ & 442 & 44.3 \\
\hline & $>8$ & 18 & 1.8 \\
\hline \multirow{3}{*}{ SES } & Low & 367 & 36.7 \\
\hline & Medium & 529 & 52.9 \\
\hline & High & 104 & 10.4 \\
\hline \multirow{2}{*}{$\begin{array}{l}\text { Involvement in } \\
\text { exercise }\end{array}$} & Yes & 121 & 12.1 \\
\hline & No & 879 & 87.9 \\
\hline
\end{tabular}

SES: Socioeconomic status.

practicing exercise. It is evident that a higher proportion of females from the overweight and obese group was involved in physical activity whereas a markedly higher percentage of the underweight group had a sedentary lifestyle. Statistically, the difference in weight indicated an extremely significant association with lifestyle.

Further, a higher percentage of high SES (74.2\%) contributed to regular physical activity. Similarly, 69.9\% of low SES had a sedentary lifestyle. Finally, no significant differences were observed among the participants in terms of age.

Table 3 represents the relationship between menstrual characteristics and physical activity involvement. As shown, short and long cycles were more prevalent among women who had a sedentary lifestyle. In addition, a mild association was observed between physical activity involvement and cycle length. Based on the obtained data, the majority of participants reported regular periods thus physically active women had an evidently higher percentage of regularity in their periods. The length and regularity of menstrual cycles reflect changes in ovarian steroid production.

The frequency of dysmenorrhea among physically active women was lower compared to those with a 
Table 2. Physical Activity Involvement Among Young Females According to BMI, SES, and Age

\begin{tabular}{|c|c|c|c|c|}
\hline Variables & & Physically Active (n=121) & Sedentary $(n=130)$ & Chi-square \\
\hline \multirow{2}{*}{ Age groups } & Adolescence & $45(46.4)$ & $52(53.6)$ & \multirow{2}{*}{$0.209^{\mathrm{NS}}$} \\
\hline & Adult & $76(49.4)$ & $78(50.6)$ & \\
\hline \multirow{3}{*}{ SES } & Low & $28(30.1)$ & 65 (69.9) & \multirow{3}{*}{$23.016^{\mathrm{a}}$} \\
\hline & Middle & $70(55.1)$ & 57 (44.9) & \\
\hline & High & $23(74.2)$ & $8(25.8)$ & \\
\hline \multirow{3}{*}{ BMI } & Underweight & $34(38.2)$ & $60(63.8)$ & \multirow{3}{*}{$9.328^{\mathrm{a}}$} \\
\hline & Normal weight & $65(53.7)$ & $56(46.3)$ & \\
\hline & Overweight \& obese & $22(61.1)$ & $14(38.9)$ & \\
\hline
\end{tabular}

a $P=0.000 ;$ NS, not significant.

BMI: Body mass index; SES: Socioeconomic status.

Table 3. Menstrual Characteristics of Physically Active and Inactive Young Females

\begin{tabular}{|c|c|c|c|c|}
\hline Variables & & Physically Active (n=121) & Sedentary $(n=130)$ & Chi-square \\
\hline \multirow{4}{*}{ Cycle length } & $<21$ days & $12(10.5)$ & $15(12.2)$ & \multirow{4}{*}{$8.815^{\mathrm{a}}$} \\
\hline & $21-27$ & $39(34.2)$ & $52(42.3)$ & \\
\hline & $28-35$ & $55(48.2)$ & 38 (30.9) & \\
\hline & $>35$ & $8(7.1)$ & $18(14.6)$ & \\
\hline \multirow{3}{*}{ Duration of flow (days) } & $\leq 4$ & $50(41.3)$ & $49(38.3)$ & \multirow{3}{*}{$0.886^{\mathrm{NS}}$} \\
\hline & $5-6$ & $61(50.4)$ & $64(50.0)$ & \\
\hline & $\geq 7$ & $10(8.3)$ & 15 (11.7) & \\
\hline \multirow{3}{*}{ Menstrual blood loss } & Scarce & $36(30.0)$ & $46(35.4)$ & \multirow{3}{*}{$1.442^{\mathrm{NS}}$} \\
\hline & Moderate & $36(30.0)$ & $31(23.8)$ & \\
\hline & Abundant & $48(40.0)$ & $53(40.8)$ & \\
\hline \multirow{2}{*}{ Regular periods } & Yes & $106(88.3)$ & $97(75.8)$ & \multirow{2}{*}{$6.570^{\mathrm{b}}$} \\
\hline & No & $14(11.7)$ & $31(24.2)$ & \\
\hline \multirow{2}{*}{ Dysmenorrhea } & Yes & $79(65.3)$ & $96(75.0)$ & \multirow{2}{*}{$2.808^{\mathrm{NS}}$} \\
\hline & No & $42(34.7)$ & $32(25.0)$ & \\
\hline \multirow{3}{*}{ Severity of dysmenorrhea } & Mild & $30(38.0)$ & $17(17.7)$ & \multirow{3}{*}{$10.832^{\mathrm{b}}$} \\
\hline & Moderate & $29(36.7)$ & $56(58.3)$ & \\
\hline & severe & $20(25.3)$ & $23(24.0)$ & \\
\hline
\end{tabular}

${ }^{\mathrm{a}} P=0.01 ;{ }^{\mathrm{b}} P=0.001 ; \mathrm{NS}$, not significant.

sedentary lifestyle (65.3\% vs $75 \%)$. However, no significant differences were found in the occurrence of dysmenorrhea $\left(\chi^{2}=2.808\right)$. Interestingly, data reveals that the severity of dysmenorrhea in the participants was closely associated with their engagement in physical activities. Further, the majority of dysmenorrheic subjects of physically active and sedentary groups $(74.7 \%$ and $76 \%)$ reported mild to moderate menstrual pain, respectively. It is worth mentioning that a higher percentage of dysmenorrheic and physically active women experienced mild pain (38\%) while higher than $58.3 \%$ of sedentary women suffered from moderate menstrual pain. Neither the duration of flow days nor the menstrual blood loss was proved to be significantly different in the two groups.

Furthermore, more than three-fourths of physically active participants highlighted staying in good shape as the predominant benefit of physical activity and it was the most frequently cited benefit in each age, BMI, and SES group.

\section{Discussion}

Although physical activity yields several advantages, a relatively limited number of women are involved in perceptible physical activity, and this exerts to Indian women as well. A small percentage of the study population used to do regular exercises (12.1\%).

However, some previous studies found that physical activity involvement was significantly and inversely associated with age $(17,18)$. while our data failed to support the findings of other studies mainly because of 
the age range of our study population. Sternfeld et al. also found a positive relationship between women's physical activity and their ages (19).

Overall, several other studies reported that higher SES determinants cause a greater rate of physical activity initiatives whereas low SES leads to low physical activity $(20,21)$.

Additionally, other studies found an association between the frequency of physical activity and SES (22, 23). In the current study, women with a higher percentage of high SES were found physically active while those with the lowest percentage of involvement in physical activity had low SES.

It is always a matter of concern that overweight and obese women are less active and it is logical to expect high rates of sedentary lifestyle among these women. Interestingly, in the present study, a higher percentage of overweight and obese females were involved in physical activity (61.1\%) and a higher percentage of underweight subjects had sedentary behavior. It could be due to multifactorial reasons such as age group, attitude toward physical activity, and weight. On the other hand, SES is a definite factor contributing to the discrepancy in body weight perception (24-26). thus it is associated with the involvement of women in the physical activity. The very fact is that being overweight made these women stick to some activities and prevalent wrong perceptions among underweight females convinced them to avoid physical activity. In populations with a lack of such awareness, the majority of people go by their perception and tend to adopt the wrong reason for the lack of physical activity.

Based on the literature reviews, there as a relationship between physical activity and menstrual function $(14,27)$. Researchers also suggested that physical activity pursuit was probably related to menstrual patterns such as the length of the cycles, menstrual regularity, the duration of bleeding, and the like.

Similarly, an association was found between the length of the cycle, regularity in periods, and the severity of dysmenorrhea with physical activity involvement in the current study.

However, there are some contradictory results regarding the relationship between cycle length and physical activity involvement in the literature (27).

Menstruation demonstrates a specific function and interplay between several endocrine glands including the hypothalamus, pituitary, and ovaries. The regularity of the cycles reflects normal reproductive activity as well (28).

Although several studies reported that there is no relationship between exercise and the regularity of menstruation, these two parameters were significantly related based on the results of the present study $(29,30)$.

Menstrual pain, which is one of the most prevalent gynecological problems is considered as a diminishing period for females. Although various researches from different parts of India have reported an increased rate of prevalence (31), the main reason for primary dysmenorrhea is still unclear (32) and non-biological factors in the etiology of primary dysmenorrhea are less known. It is also believed that the frequency of primary dysmenorrhea reduces by doing exercise. Likewise, previous research revealed that the prevalence of primary dysmenorrhea and other menstrual symptoms is related to exercise (33).

According to a review study, it seems there is no relationship between dysmenorrhea and exercise/physical activity patterns when the number of participants is more than 500 while studies on small-scale population $(<500$ participants), where the bias is also more likely to be present, report positive associations. Contrarily, other studies found no significant association in this respect. Our results did not support the relationship between exercise and the prevalence of primary dysmenorrhea as well (33-35).

However, exercise seems to improve the blood flow at the pelvic and provokes the release of endorphins which function as non-specific analgesics (36). Our results supported the hypothesis that those women with sedentary lifestyle indicate higher levels of dysmenorrhea compared to physically active women. In similar studies conducted in Iran, researchers found the very significant role of physical activity in reducing the severity of dysmenorrhea and premenstrual syndrome $(37,38)$, which is in line with our findings. However, there are some controversial reports as well. Menstrual differences between the groups might not be relevant probably due to the moderate intensity of physical exercise in the group who were physically active.

\section{Conclusions}

In general, the present study obtained considerable results because, to the best of our knowledge, no study has so far well-explored and understood physical activity involvement. It is important to understand the relationship between involvement in physical activity, subjective factors, and menstrual characteristics. Given that a proper understanding of physical activity can be effective in the health and life of young women, it is essential to study the prevalence of involvement in physical activity and its correlates. In this regard, there is a need for evaluating the factors which influence lifestyle behaviors including physical activity among Indian females. Thus, further investigation is warranted in this respect.

\section{Conflict of Interests}

Authors declare that they have no conflict of interests.

\section{Ethical Issues}

Not applicable.

\section{Financial Support}

None. 


\section{Acknowledgments}

The authors are grateful to all participants.

\section{References}

1. Strong WB, Malina RM, Blimkie CJ, et al. Evidence based physical activity for school-age youth. J Pediatr. 2005;146(6):732-737. doi:10.1016/j.jpeds.2005.01.055

2. Riddoch CJ, Bo Andersen L, Wedderkopp N, et al. Physical activity levels and patterns of 9- and 15-yr-old European children. Med Sci Sports Exerc. 2004;36(1):86-92. doi:10.1249/01.mss.0000106174.43932.92

3. Prevalence of regular physical activity among adults-United States, 2001 and 2005. MMWR Morb Mortal Wkly Rep. 2007;56(46):1209-1212.

4. Allison MJ, Keller C. Physical activity maintenance in elders with cardiac problems. Geriatr Nurs. 2000;21(4):200-203. doi:10.1067/mgn.2000.101100

5. Fahrenwald NL, Walker SN. Application of the Transtheoretical Model of behavior change to the physical activity behavior of WIC mothers. Public Health Nurs. 2003;20(4):307-317.

6. Juarbe TC, Lipson JG, Turok X. Physical activity beliefs, behaviors, and cardiovascular fitness of Mexican immigrant women. J Transcult Nurs. 2003;14(2):108-116. doi:10.1177/1043659602250623

7. Ourania M, Yvoni H, Christos K, Ionannis T. Effects of a physical activity program. The study of selected physical abilities among elderly women. J Gerontol Nurs. 2003;29(7):50-55.

8. Nies MA, Chruscial HL. Neighborhood and physical activity outcomes in women: regional comparisons. Nurs Clin North Am. 2002;37(2):295-301, vii.

9. Taylor L, Whittington F, Hollingsworth C, et al. Assessing the effectiveness of a walking program on physical function of residents living in an assisted living facility. J Community Health Nurs. 2003;20(1):15-26. doi:10.1207/ s15327655jchn2001_02

10. United States. Public Health Service. Office of the Surgeon General President's Council on Physical Fitness Sports Physical activity and health: a report of the Surgeon General. Jones \& Bartlett Learning; 1998.

11. Pate RR, Pratt M, Blair SN, et al. Physical activity and public health. A recommendation from the Centers for Disease Control and Prevention and the American College of Sports Medicine. Jama. 1995;273(5):402-407.

12. Blair SN, LaMonte MJ, Nichaman MZ. The evolution of physical activity recommendations: how much is enough? Am J Clin Nutr. 2004;79(5):913s-920s. doi:10.1093/ ajcn/79.5.913S

13. Henderson KA, Ainsworth BE. A synthesis of perceptions about physical activity among older African American and American Indian women. Am J Public Health. 2003;93(2):313-317. doi:10.2105/ajph.93.2.313

14. Liu Y, Gold EB, Lasley BL, Johnson WO. Factors affecting menstrual cycle characteristics. Am J Epidemiol. 2004;160(2):131-140. doi:10.1093/aje/kwh188

15. Revill SI, Robinson JO, Rosen M, Hogg MI. The reliability of a linear analogue for evaluating pain. Anaesthesia. 1976;31(9):1191-1198.

16. Higham JM, O'Brien PM, Shaw RW. Assessment of menstrual blood loss using a pictorial chart. Br J Obstet Gynaecol. 1990;97(8):734-739.

17. Laffrey SC. Physical activity among older Mexican American women. Res Nurs Health. 2000;23(5):383-392.

18. Duffy ME. Determinants of reported health promotion behaviors in employed Mexican American women. Health Care Women Int. 1997;18(2):149-163. doi:10.1080/07399339709516270

19. Sternfeld B, Cauley J, Harlow S, Liu G, Lee M. Assessment of physical activity with a single global question in a large, multiethnic sample of midlife women. Am J Epidemiol. 2000;152(7):678-687. doi:10.1093/aje/152.7.678

20. Bradley CB, McMurray RG, Harrell JS, Deng S. Changes in common activities of 3rd through 10th graders: the CHIC study. Med Sci Sports Exerc. 2000;32(12):2071-2078.

21. Gordon-Larsen P, McMurray RG, Popkin BM. Determinants of adolescent physical activity and inactivity patterns. Pediatrics. 2000;105(6):E83. doi:10.1542/peds.105.6.e83

22. Lampert T, Mensink GB, Romahn N, Woll A. [Physical activity among children and adolescents in Germany. Results of the German Health Interview and Examination Survey for Children and Adolescents (KiGGS)]. Bundesgesundheitsblatt Gesundheitsforschung Gesundheitsschutz. 2007;50(5-6):634-642. doi:10.1007/s00103-007-0224-8

23. Finne E, Bucksch J, Lampert T, Kolip P. Age, puberty, body dissatisfaction, and physical activity decline in adolescents. Results of the German Health Interview and Examination Survey (KiGGS). Int J Behav Nutr Phys Act. 2011;8:119. doi:10.1186/1479-5868-8-119

24. Paeratakul S, White MA, Williamson DA, Ryan DH, Bray GA. Sex, race/ethnicity, socioeconomic status, and BMI in relation to self-perception of overweight. Obes Res. 2002;10(5):345-350. doi:10.1038/oby.2002.48

25. Sarlio-Lahteenkorva S, Silventoinen K, Lahelma E. Relative weight and income at different levels of socioeconomic status. Am J Public Health. 2004;94(3):468-472. doi:10.2105/ ajph.94.3.468

26. Kuchler F, Variyam JN. Mistakes were made: misperception as a barrier to reducing overweight. Int J Obes Relat Metab Disord. 2003;27(7):856-861. doi:10.1038/sj.ijo.0802293

27. Sternfeld B, Jacobs MK, Quesenberry CP Jr, Gold EB, Sowers M. Physical activity and menstrual cycle characteristics in two prospective cohorts. Am J Epidemiol. 2002;156(5):402409. doi:10.1093/aje/kwf060

28. Roupas ND, Georgopoulos NA. Menstrual function in sports. Hormones (Athens). 2011;10(2):104-116. doi:10.14310/horm.2002.1300

29. Chang JS. Refashioning womanhood in 1990s Taiwan: An analysis of the Taiwanese edition of Cosmopolitan magazine. Mod China. 2004;30(3):361-397.

30. Casper RC, Michaels J, Simon K. Body perception and emotional health in athletes: a study of female adolescents involved in aesthetic sports. World Rev Nutr Diet. 1997;82:134-147.

31. Agarwal AK, Agarwal A. A study of dysmenorrhea during menstruation in adolescent girls. Indian J Community Med. 2010;35(1):159-164. doi:10.4103/0970-0218.62586

32. Davis AR, Westhoff CL. Primary dysmenorrhea in adolescent girls and treatment with oral contraceptives. J Pediatr Adolesc Gynecol. 2001;14(1):3-8.

33. Daley AJ. Exercise and primary dysmenorrhoea: a 
comprehensive and critical review of the literature. Sports Med. 2008;38(8):659-670. doi:10.2165/00007256200838080-00004

34. Latthe P, Mignini L, Gray R, Hills R, Khan K. Factors predisposing women to chronic pelvic pain: systematic review. Bmj. 2006;332(7544):749-755. doi:10.1136/ bmj.38748.697465.55

35. Blakey H, Chisholm C, Dear F, et al. Is exercise associated with primary dysmenorrhoea in young women? BJOG. 2010;117(2):222-224. doi:10.1111/j.14710528.2009.02220.x
36. Proctor M, Farquhar C. Diagnosis and management of dysmenorrhoea. Bmj. 2006;332(7550):1134-1138. doi:10.1136/bmj.332.7550.1134

37. Koushkie Jahromi, M, Gaeini A, Rahimi Z. Influence of a physical fitness course on menstrual cycle characteristics. Gynecol Endocrinol. 2008;24(11):659-662. doi:10.1080/09513590802342874

38. Safarzadeh A, Zare S, Arbabisarjou A, Ghoreishinia G. The relationship between Exercise and premenstrual syndrome. International Journal of Medical Research \& Health Sciences. 2016;5(9):183-189.

(C) 2019 The Author (s); This is an open-access article distributed under the terms of the Creative Commons Attribution License (http://creativecommons.org/licenses/by/4.0), which permits unrestricted use, distribution, and reproduction in any medium, provided the original work is properly cited. 